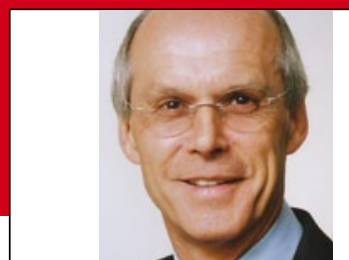

Prof. Dr. med. H. S. FüeßI Isar-AmperKlinikum, KI. München-Ost, Haar

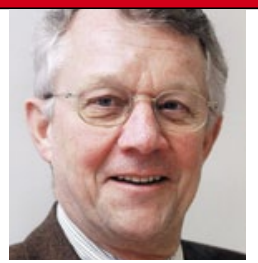

Prof. Dr. med. H. Holzgreve Internist, Kardiologische Praxis, München

\title{
Erhöhte Suizidrate nach Beginn der Finanzkrise
}

Mit dem Beginn der Finanzkrise hat die Selbstmordrate in England deutlich zugenommen. Sie korreliert mit dem Grad der Arbeitslosigkeit in einzelnen Regionen.

— Die jährlichen Raten von Suiziden und tödlichen Unfällen unklarer Ursache für die Jahre 2000 bis 2012 und für 93 Regionen in England wurden den nationalen Datenbanken entnommen. Gleichzeitig wurden die Anträge für Arbeitslosengeld in den einzelnen Regionen eruiert.

In den Jahren 2000 bis 2007, also vor Beginn der Finanzkrise, sank die Zahl der Suizide bei Männern um 57, bei Frauen um 26 pro Jahr. Unter der Annahme, dass dieser längjährige Trend sich fortsetzt, errechneten die Autoren für den Zeitraum von 2008 bis 2010 einen Anstieg um 846 Suizide bei Männern und 155 bei Frauen. Die Suizidraten bei beiden Geschlechtern korrelieren signifikant $(\mathrm{P}<0,001)$ mit der Zahl der Arbeitslosen (s. Abb.). Regionen mit stärkerer Arbeitslosigkeit haben auch höhere Suizidraten.

In einer weiteren Analyse überprüften die Autoren die Auswirkungen der Kurzzeitarbeitslosigkeit unter Ausschluss von Langzeitarbeitslosen. In den
Jahren zwischen 2000 und 2010 führte eine Zunahme der Arbeitslosigkeit um $10 \% \mathrm{zu}$ einem Anstieg der Suizidrate um $1,4 \%$ bei Männern und um $0,7 \%$ bei Frauen. Zwischen 2008 und 2010 stieg die Arbeitslosigkeit jährlich um 25,6\%. Aus weiteren Analysen leiten die Autoren ab, dass mindestens 2/5 aller Suizide auf die Arbeitslosigkeit zurückzuführen sind.

\footnotetext{
- N. Barr et al.

Suicides associated with the 2008-10 economic recession in England: time trend analysis. Brit. Med. 2012;345:e5142
}

\section{Abbildung 1}

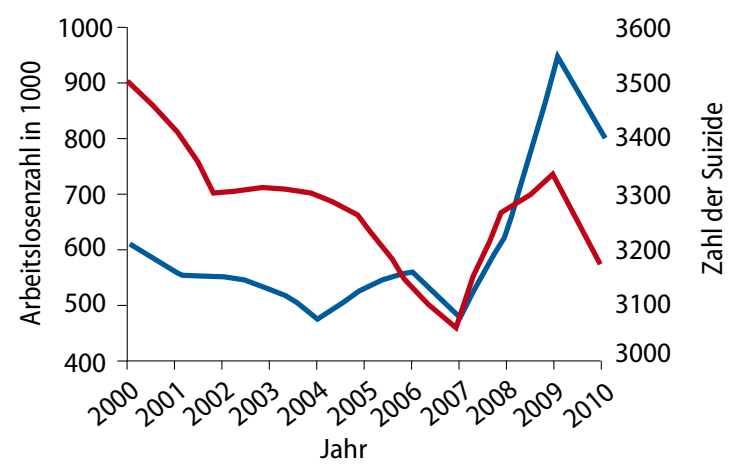

Trends für die Zahl der Arbeitslosen (blau, links) und der Zahl der Suizide (rot) bei Männern in den Jahren 2000 bis 2010.

\section{Kommentar}

Die Autoren diskutierten zahlreiche Aspekte und Schwächen der Ergebnisse. Schlussendlich machen sie aber doch aufgrund der letztlich überzeugenden zeitlichen Korrelation die Folgen der Finanzkrise, insbesondere den Anstieg der Arbeitslosigkeit für die deutliche Zunahme der Suizide verantwortlich. Wenn ein solches Thema in einer renommierten medizinischen Fachzeitschrift publiziert wird, erwartet man eigentlich eine Diskussion zu medizinischen und ärztlichen Aspekten und Konsequenzen, z. B. zur Früherkennung und Prävention von Suiziden in einer bedrohten Patientenklientel. Doch die Diskussion hat eine höchst ungewöhnliche, rein politische Stoßrichtung: Die Autoren geißeln die Sparmaßnahmen, die Etatkürzungen, die Lockerungen des Kündigungsschutzes usw. als Ursache der Suizide. Die Entscheidungen der Politiker werden als falsch bezeichnet und für diese Entwicklung verantwortlich gemacht.

H. HOLZGREVE . 